\title{
Correction to: Effect of Opioid Use on Immune Activation and HIV Persistence on ART
}

\author{
Livio Azzoni ${ }^{1}$ - David Metzger ${ }^{2}$ - Luis J. Montaner ${ }^{1}$ (D) \\ Published online: 24 October 2020 \\ (C) Springer Science+Business Media, LLC, part of Springer Nature 2020
}

\section{Correction to: Journal of Neuroimmune Pharmacology https://doi.org/10.1007/s11481-020-09959-y}

The original version of this article unfortunately contained mistakes.

\section{1. abstract first sentence is missing a "of"}

While there is an emerging consensus that engagement of the $\mathrm{Mu}$ opioid receptor by opioids may modulate various stages the HIV life cycle (e.g.: increasing cell susceptibility to infection, promoting viral transcription, and depressing immune responses to virally-infected cells), the overall effect on latency and viral reservoirs remains unclear.

should read

While there is an emerging consensus that engagement of the $\mathrm{Mu}$ opioid receptor by opioids may modulate various stages of the HIV life cycle (e.g.: increasing cell susceptibility to infection, promoting viral transcription, and depressing immune responses to virallyinfected cells), the overall effect on latency and viral reservoirs remains unclear.

The online version of the original article can be found at https://doi.org/ 10.1007/s11481-020-09959-y

Luis J. Montaner

montaner@wistar.org

1 HIV Immunopathogenesis Laboratory, The Wistar Institute, 3601 Spruce Street, Philadelphia, PA 19104, USA

2 Department of Psychiatry, University of Pennsylvania Perelman School of Medicine, 3535 Market Street, Suite 4100,

Philadelphia, PA 19104, USA

2. Section heading has an extra "s":

Effect of Opioids on HIV Infection: Synergistics Immune Modulation

should read

Effect of Opioids on HIV Infection: Synergistic Immune Modulation

3. Section heading is missing a ":"

Opioids and Persistent HIV Reservoirs

Dynamics on ART

should read

Opioids and Persistent HIV Reservoirs:

Dynamics on ART

4. Section heading has an added "S"

Future Directions: Opioids and HIV Reservoirs Dynamics

should read

Future Directions: Opioids and HIV Reservoir Dynamics

Publisher's Note Springer Nature remains neutral with regard to jurisdictional claims in published maps and institutional affiliations. 